\title{
Revisión
}

\section{Guía del carcinoma de células renales}

\author{
Börje Ljungberga, Damian C. Hanbury ${ }^{\mathrm{b}}$, Marcus A. Kuczyk ${ }^{\mathrm{c}}$, Axel S. Merseburger ${ }^{\mathrm{c}}$, \\ Peter F.A. Mulders ${ }^{\mathrm{d}}$, Jean-Jacques Patard ${ }^{\mathrm{e}}$, Ioanel C. Sinescu ${ }^{\mathrm{f}}$
}

\author{
aServicio de Cirugía y Ciencias Perioperatorias, Urología y Andrología, Universidad de Umea, Umea, Suecia. \\ ${ }^{b}$ Servicio de Urología, Hospital de Lister, Mill Lane, Stevenage, Reino Unido. \\ 'Servicio de Urología, Universidad de Tuebingen, Tuebingen, Alemania. \\ dServicio de Urología, Centro Médico de la Universidad Radbound, Nijmegen, Holanda. \\ eServicio de Urología, CHU Pontchaillou, Rennes, Francia.

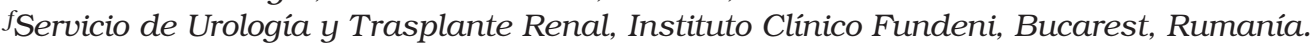

Traducción del artículo european uRology 51 (2007) 1502-1510: Gonzalo García Fadrigue

Servicio de Urología. Hospital Universitario La Fe. Valencia, España.

\section{Resumen}

Objetivos: El Grupo de trabajo de la Asociación Europea de Urología (AEU) sobre el Carcinoma de Células Renales (CCR) ha preparado esta guía para ayudar a los urólogos a evaluar la evidencia sobre el manejo del CCR y a incorporar sus recomendaciones a su práctica clínica.

Material y métodos: Las recomendaciones proporcionadas en esta guía están basadas en una búsqueda sistemática de la literatura utilizando Medline, el Registro Central de Ensayos Controlados de la Cochrane, así como en publicaciones y artículos de revisión.

Resultados: Hay un número limitado de estudios prospectivos aleatorizados con alto nivel de evidencia. La mayoría de las publicaciones acerca del CCR se basan en análisis retrospectivos, incluyendo algunos estudios multicéntricos más largos validados y estudios controlados bien diseñados.

Conclusiones: se debe remarcar que esta guía contiene información para el tratamiento individual de un paciente de acuerdo a un enfoque general estándar. El manejo clínico de los pacientes con CCR puede mejorarse con recomendaciones actualizadas sobre diagnóstico, tratamiento y seguimiento.

Palabras claves: Diagnóstico. Seguimiento. Recomendaciones. Guías. Carcinoma de células renales. Tratamiento

\section{Renal cell carcinoma guideline}

\section{Abstract}

Objectives:The European Association of Urology (EAU) Guideline Group for renal cell carcinoma (RCC) prepared this guideline to help urologists assess the evidence-based management of RCC and to incorporate the guideline recommendations into their clinical practice.

Methods:The re commendations provided in the current guideline are based on a systematic literature search using MedLine, the Cochrane Central Register of Controlled Trials, and publications and review articles.

Results: A Limited Number of prospective randomized studies are available with high-level evidence.Most publications concerning RCC are based on retrospective analyses, including some larger multicentre validation studies and well-designed controlled studies.

Conclusions: It must be stressed that the current guideline contains information for the treatment of an individual patient according to a standardized general approach. Updated recommendations concerning diagnosis, treatment, and follow-up can improve the clinical handling of patients with RCC.

Keywords: Diagnosis. Follow-up. Recommendations. Guidelines. Renal cell carcinoma. Treatment.

\section{Introducción}

El Grupo de trabajo de la Asociación Europea de Urología (AEU) sobre el Carcinoma de Células Renales (CCR) ha preparado esta guía para ayudar a los urólogos a evaluar la evidencia sobre el manejo del CCR y a incorporar sus recomendaciones a su práctica clínica. Se puede encontrar información detallada sobre el nivel de evidencia y el grado de recomendación en: www.uroweb.nl. Las publicaciones referentes al CCR se basan sobretodo en análisis

(*)La traducción de este artículo se ha llevado a cabo con el permiso de la Asociación Europea de Urología. 
retrospectivos, incluyendo algunos estudios multicéntricos más largos validados y estudios controlados bien diseñados. Sólo se dispone de unos pocos estudios prospectivos aleatorizados con elevado nivel de evidencia. Por lo tanto es difícil conseguir información basada en la evidencia. Las recomendaciones proporcionadas en esta guía están basadas en una búsqueda sistemática de la literatura utilizando Medline, el Registro Central de Ensayos Controlados de la Cochrane, así como en publicaciones y artículos de revisión. Se debe remarcar que esta guía contiene información para el tratamiento individual de un paciente de acuerdo a un enfoque general estándar. La información se debe tener en cuenta como recomendaciones sin implicaciones legales.

\section{Trasfondo: epidemiologia y etiologia}

El CCR representa el 2-3\% de todos los tumores $^{1}$, con una mayor incidencia en los países desarrollados. El incremento anual de la incidencia tanto en Europa como en todo el mundo es de aproximadamente un 2\%, aunque en Dinamarca y Suecia se ha observado un descenso continuado durante las últimas dos décadas ${ }^{2}$. En 1998, cerca de 30.000 pacientes fueron diagnosticados de cáncer renal en la Unión Europea, y aproximadamente 15.000 murieron de esta enfermedad ${ }^{1}$.

El CCR es la lesión sólida más frecuente en el riñón y comprende diferentes tipos, con características histopatológicas y genéticas específicas ${ }^{3}$. Hay un predominio de 1,5:1 de los hombres sobre las mujeres, con un pico de incidencia entre los 60 y los 70 años de edad. Entre los factores etiológicos se incluyen algunos del estilo de vida, como el tabaquismo, la obesidad y la hipertensión ${ }^{2,4}$. El consumo de cigarrillos es un factor de riesgo definido para el CCR. Queda por clarificar definitivamente el papel de la obesidad y la hipertensión como factores de riesgo para el CCR. La medida preventiva más importante para el CCR es abandonar el consumo de cigarrillos.

Debido al incremento en la detección de tumores por el uso de técnicas de imagen como la ecografía y la tomografía computarizada (TC), se encuentran un número creciente de CCR incidentales. Estos tumores son con mayor frecuencia menores y de estadios más bajos ${ }^{5,6}$. A pesar del incremento en la detección incidental, la mortalidad por CCR se ha mantenido sin modificaciones y paralela a la incidencia.

\section{Diagnóstico y estadificación \\ 3.1 Síntomas}

Muchas masas renales permanecen asintomáticas y no palpables hasta bien avanzado el curso natural de la enfermedad. En la actualidad, más del 50\% de los CCR se detectan de manera incidental al utilizar técnicas de imagen no invasivas para evaluar múltiples síntomas inespecíficos ${ }^{7}$. La tríada clásica de dolor en el flanco, hematuria macroscópica y masa abdominal palpable se encuentra hoy en día raramente $(6-10 \%)^{8,9}$. Se encuentran síndromes paraneoplásicos en alrededor del 30\% de los pacientes con CCR sintomático. Los más comunes son hipertensión, caquexia, pérdida de peso, pirexia, neuromiopatía, amiloidosis, elevación de la velocidad de sedimentación globular, anemia, disfunción hepática, hipercalcemia, policitemia, etc ${ }^{7}$. Una minoría de los pacientes debuta con síntomas directamente causados por la enfermedad metastásica, como dolor óseo o tos persistente ${ }^{7}$. Todavía, el 25$30 \%$ de los pacientes se diagnostican por síntomas asociados a enfermedad metastásica.

La exploración física tiene un papel limitado en el diagnóstico del CCR, pero es valiosa en algunos pacientes, como aquellos con una masa abdominal palpable, adenopatías cervicales palpables, varicocele irreductible o edema bilateral en los miembros inferiores, que sugiere afectación venosa. Los parámetros analíticos más frecuentemente evaluados son la hemoglobina, la velocidad de sedimentación globular, la fosfatasa alcalina y el calcio sérico ${ }^{7,10}$.

\subsection{Exámenes radiológicos}

La mayoría de los tumores renales se diagnostican por una ecografía abdominal o una TC realizados por diversos motivos. El hallazgo ecográfico de una masa renal sólida debe ser investigado más profundamente mediante un TC de alta resolución con medio de contraste. El gold estándar del diagnóstico del CCR, el TC helicoidal, evalúa el tumor primario, su extensión extrarrenal y proporciona información sobre la afectación venosa, crecimiento de adenopatías locorregionales, estado de las glándulas suprarrenales y del hígado, y del funcionamiento y morfología del riñón contralateral. A pesar de que la TC torácica es la prueba más precisa para estadificar el tórax ${ }^{11}$, como mínimo una radiografía del tórax (como alternativa menos precisa) debe realizarse de rutina para evaluar la existencia de metástasis. La Resonancia Magnética (RM) se puede 
reservar fundamentalmente para los pacientes con enfermedad localmente avanzada, sospecha de afectación venosa, insuficiencia renal o alergia al contraste intravenoso ${ }^{12}$. La RM es también una opción para evaluar la extensión por la vena cava del trombo tumoral, así como para valorar masas renales no filiadas. La ecografía doppler y la ecografía transesofágica son útiles también para valorar el trombo tumoral $^{13}$. Otras pruebas diagnósticas, como el rastreo óseo, TC cerebral o RM, pueden aplicarse si los síntomas clínicos o datos de laboratorio así lo requieren. La arteriografía renal, la cavografía inferior o la punción biopsia con aguja fina tienen un papel limitado en el estudio clínico de los pacientes con CCR, pero en casos seleccionados se deben considerar.

En resumen, se recomienda que en un paciente con al menos uno de estos hallazgos clínicos o de laboratorio se debe sospechar la presencia de un CCR. Una radiografía simple de tórax puede ser suficiente para evaluar los pulmones en pacientes de bajo riesgo, pero la TC torácica es más sensible. La TC abdominal y RM se recomiendan para el estudio de los pacientes con CCR y son las pruebas de imagen más adecuadas para realizar la estadificación TNM previamente a la cirugía.

\subsection{Clasificación y pronóstico}

Para la estadificación del CCR se recomienda la clasificación TNM de 2002 de la UICC ${ }^{14}$. No está claro que la actual clasificación TNM sea óptima para predecir la supervivencia en pacientes con CCR y podría ser reclasificada. La subdivisión del estadio pTa, introducida en $2002^{14}$, ha sido validada con numerosos estudios ${ }^{15}$. No obstante, se deben hacer mejoras en la clasificación de los tumores pT3. En primer lugar, no se ha clarificado si cuando existe solamente invasión de la grasa del seno renal el pronóstico es el mismo que cuando hay invasión de la grasa perinefrítica ${ }^{16}$. En segundo lugar, se ha sugerido que los CCR con invasión de la glándula suprarrenal deberían clasificarse como T4 ${ }^{17}$. Además, aún no está claro si la estratificación de los CCR con invasión venosa en T3b y T3c es exacta. Se requieren más estudios para investigar el valor pronóstico independiente de la invasión de la vena cava comparada con la invasión de la vena renal. Recientemente se ha cuestionado la precisión de la subclasificación de N1-N2 ${ }^{18}$. Por último, para una correcta estadificación $\mathrm{M}$ de los pacientes con CCR, se debe realizar una técnica de imagen precisa como un TC toracoabdominal.

Los factores con implicación pronóstica se clasifican en anatómicos, histológicos, clínicos y moleculares. Los factores anatómicos incluyen el tamaño tumoral, la invasión venosa, la invasión de la cápsula renal, la afectación adrenal y la metástasis ganglionar o a distancia. Estos factores frecuentemente se agrupan en la clasificación TNM de 2002.

Los factores pronósticos histológicos incluyen el grado de Fuhrman, el subtipo histológico, la presencia de componente sarcomatoide, la invasión microscópica de la pared venosa, la necrosis tumoral y la invasión de los ductos colectores. El grado nuclear de Fuhrman es el sistema de gradación histológica más ampliamente aceptado en el CCR. A pesar de tener variaciones intra e interobservador constituye un factor pronóstico independiente. De acuerdo con la clasificación de la Organización Mundial de la Salud (OMS) ${ }^{19}$,existen tres subtipos histológicos principales de CCR: convencional (células claras, 80-90\%), papilar (10-15\%), y cromófobo (4-5\%). Numerosos estudios han demostrado que tienen mejor pronóstico los pacientes con CCR cromófobo, papilar y convencional, respectivamente. Sin embargo, la información pronóstica del subtipo histológico de CCR se pierde al clasificar a los tumores en estadios. Dentro de los CCR papilares se han identificado dos subgrupos con diferente evolución ${ }^{20}$. Los tumores Tipo I son de bajo grado, con citoplasma cromofílico y un pronóstico favorable. Los CCR papilares de Tipo II son generalmente de alto grado, con citoplasma eosinofílico y mayor tendencia a desarrollar metástasis. Esta subclasificación del CCR se ha confirmado mediante estudios moleculares y citogenéticos.

Entre los factores clínicos se incluye el estado general del paciente, sintomas locales, caquexia, anemia y cifra de plaquetas. Se están investigando numerosos marcadores moleculares como la anhidrasa carbónica IX (CAIX), el factor de crecimiento del endotelio vascular (VEGF), el factor hipoxiainducible (HIF), Ki67 (proliferación), p53, PTEN (ciclo celular), E cadherinas y CD44 (adhesión celular). Estos marcadores todavía no son de uso extendido. Recientemente se han identificado 259 genes con implicación pronóstica en cuanto a supervivencia independiente de los factores clínicos en el CCR convencional, lo que indica que la información genética mejorará el pronóstico ${ }^{21}$. 
Se recomienda utilizar el actual sistema de clasificación TNM, el grado de Fuhrman y el subtipo histológico de CCR, porque tienen valor para el pronóstico y para decidir el tratamiento. No se recomienda de forma rutinaria el uso de nomogramas pronósticos, aunque pueden ser útiles para determinar el pronóstico de los pacientes de cara a incluirlos en ensayos clínicos. Se ha sugerido que estos nomogramas son más precisos que el estadio TNM o el grado de Fuhrman para predecir la supervivencia. Actualmente no existe ningún marcador molecular con valor pronóstico cuyo uso se recomiende de forma rutinaria en la práctica clínica.

\section{Tratamiento}

\subsection{Tratamiento de la enfermedad localizada}

La nefrectomía radical, incluyendo el riñón afecto, continúa siendo el único tratamiento curativo en los pacientes con CCR localizado, y ofrece una oportunidad razonable de curación. No hay evidencia a favor de un abordaje quirúrgico específico (Tabla 1$)^{22}$.

Para los tumores renales pequeños el gold estándar de tratamiento ya no incluye la adrenalectomía, como recomendaba Robson. La resección de la glándula suprarrenal no se recomienda si ésta es normal en la TC preoperatoria, y si se descarta la invasión directa de la misma por un tumor del polo superior ${ }^{23,24}$.

La linfadenectomía se debería limitar a la región perihiliar y con fines de estadificación, ya que la linfadenectomía amplia no parece mejorar la supervivencia. Los CCR con un trombo tumoral a nivel de la vena renal o de la vena cava inferior tienen un mayor grado y estadio, y mayor tendencia a desarrollar metástasis ganglionares. El pronóstico clínico viene determinado por la mayor agresividad biológica inducida por el crecimiento del tumor local- mente, junto con la presencia de metástasis a nivel de los ganglios regionales, más que por la presencia de trombo a nivel de la cava o por la extensión craneal del mismo. En los pacientes sin enfermedad metastásica se recomienda, junto con la nefrectomía, la trombectomía ${ }^{25}$.

La embolización del tumor primario está indicada en pacientes con hematuria macroscópica o síntomas locales como dolor ${ }^{26}$. Puede resultar beneficiosa también en los pacientes con malas condiciones físicas para la resección del tumor primario, así como previa a la resección quirúrgica de grandes metástasis óseas. No existe beneficio en realizar la embolización de forma rutinaria previamente a la nefrectomía radical.

\subsection{Cirugia conservadora}

Las indicaciones absolutas de la nefrectomía parcial son la existencia de un riñón único anatómico o funcional, y la existencia de un CCR bilateral. Entre las indicaciones relativas se incluye la presencia de un riñón contralateral funcional pero afectado por una dolencia que pueda limitar su función en el futuro, así como en pacientes con formas hereditarias de CCR con un alto riesgo de desarrollar un tumor en el riñón contralatera ${ }^{27,28}$.

La principal indicación electiva es un CCR unilateral localizado, con un riñón contralateral sano. Se recomienda la cirugía conservadora del órgano a los pacientes con tumores de menos de $4 \mathrm{~cm}$ de diámetro, ya que les proporciona similares tasas de supervivencia total y libre de recidiva que a los pacientes sometidos a nefrectomía radical ${ }^{29,30}$. Se han descrito resultados oncológicos similares a los de una cirugía radical en pacientes con tumores de hasta 7 cm de diámetro ${ }^{31}$. Sin embargo, la cirugía conservadora del órgano no se puede recomendar como tra-

Tabla 1. Tratamiento quirúrgico primario del carcinoma de células renales según los estadios T (TNM 2002)

\begin{tabular}{|c|c|c|c|}
\hline \multirow[t]{2}{*}{ T1a } & Cirugía conservadora del órgano & $\begin{array}{l}\text { Abierta } \\
\text { Laparoscópica }\end{array}$ & $\begin{array}{l}\text { Tratamiento estándar recomendado } \\
\text { Optativo en centros experimentados }\end{array}$ \\
\hline & Nefrectomía radical & & Razonable en pacientes seleccionados \\
\hline \multirow[t]{3}{*}{ T1b-T2 } & Nefrectomía radical & Abierta & Adecuado (alta morbilidad) \\
\hline & & Laparoscópica & Tratamiento estándar recomendado \\
\hline & Cirugía conservadora del órgano & & Factible pero generalmente no recomendado \\
\hline \multirow[t]{2}{*}{ T3, T4 } & Nefrectomía radical & Abierta & $\begin{array}{l}\text { Tratamiento estándar recomendado para la } \\
\text { mayoría de los pacientes }\end{array}$ \\
\hline & & Laparoscópica & Factible en pacientes seleccionados \\
\hline
\end{tabular}


tamiento estándar en esos casos. Si el tumor es resecado completamente, el grosor del margen quirúrgico (mayor de $1 \mathrm{~mm}$ ) no se correlaciona con la probabilidad de recidiva local. Si el CCR de gran tamaño se trata con cirugía conservadora del órgano, se debe intensificar el seguimiento debido a que existe un mayor riesgo de recidiva intrarrenal.

\subsection{Nefrectomía laparoscópica}

La nefrectomía radical laparoscópica se ha convertido en una intervención quirúrgica consolidada, con una menor morbilidad que la cirugía abierta ${ }^{32}$. El abordaje laparocópico duplica los principios oncológicos de la cirugía abierta consolidada, incluyendo el control precoz de las venas renales antes de la manipulación del tumor, una amplia manipulación de la pieza por fuera de la fascia de Gerota, la evitación del daño o ruptura de la pieza y la extracción de la pieza intacta. Actualmente, la nefrectomía radical laparoscópica se recomienda como tratamiento de referencia para los pacientes con un CCR en estadio $\mathrm{T} 1-2$, y los resultados indican tasas de supervivencia sin cáncer equivalentes a las registradas utilizando la cirugía radical abierta. Es de esperar que la nefrectomía radical laparoscópica se convierta en una modalidad de tratamiento ampliamente disponible y que los centros especializados en cáncer renal la fomenten.

En determinados pacientes y realizada por un experto, la nefrectomía parcial laparoscópica es una alternativa a la cirugía abierta ${ }^{33}$. La indicación óptima es en los pacientes con un tumor pequeño y periférico. A pesar de que se ha sugerido que los resultados oncológicos de la nefrectomía parcial laparoscópica duplican a los de la cirugía abierta, los estudios más amplios muestran que no existen datos fiables a largo plazo. Las desventajas del abordaje laparoscópico son el mayor periodo de isquemia caliente y las mayores complicaciones intra y postoperatorias que en caso de la cirugía abierta. Por lo tanto la nefrectomía parcial abierta continúa siendo actualmente el tratamiento estándar. La nefrectomía parcial laparoscópica se debe reservar a los centros experimentados.

\subsection{Tratamiento alternativo minimamente invasivo}

Las técnicas mínimamente invasivas como radiofrecuencia percutánea, crioterapia, microondas y ablación focal por ultrasonidos de alta intensidad
(HIFU) se han propuesto como posibles alternativas al tratamiento quirúrgico del CCR. Las potenciales ventajas de estas técnicas incluyen una reducción de la morbilidad, la terapia en consulta externa y la posibilidad de tratar a pacientes de alto riesgo con una salud precaria para soportar la cirugía convencional ${ }^{34,35}$. Por tanto, estos tratamientos experimentales se pueden recomendar en pacientes seleccionados con pequeñas lesiones corticales halladas de forma incidental, en pacientes ancianos, en pacientes con predisposición para tener tumores múltiples o en pacientes con un solo riñón o con tumores bilaterales. Los estudios clínicos deberán evaluar la tasa de éxito oncológico y las complicaciones posteriores a estas intervenciones.

\subsection{Terapia adyuvante}

La evidencia actual sugiere que la vacuna tumoral adyuvante puede mejorar la supervivencia libre de progresión en determinados subgrupos de pacientes sometidos a nefrectomía con cáncer renal en estadio T3, pero se necesita más investigación sobre el impacto en la supervivencia total ${ }^{36}$. Los algoritmos pronósticos pueden identificar a los pacientes que se beneficiarán en mayor medida de la terapia adyuvante con vacuna. Sin embargo, la terapia adyuvante con citocinas no mejora la supervivencia tras la nefrectomía ${ }^{37}$. Exceptuando los estudios clínicos controlados, no existen indicaciones de terapia adyuvante postquirúrgica. El éxito de los nuevos inhibidores de la tirosin-kinasa en el tratamiento del CCR metastásico ha dado pie a la recomendación de participar en ensayos clínicos con pacientes afectos de CCR con riesgo.

\section{Tratamiento del CCR metastásico 5.1 Tratamiento quirúrgico del $\mathrm{CCR}$ metastásico}

La nefrectomía es curativa sólo si la cirugía puede extraer todos los focos tumorales. En un metanálisis de dos estudios aleatorizados incluyendo sólo pacientes con un buen estado general, comparando la nefrectomía combinada con interferón- $\alpha$ (IFN- $\alpha$ ) frente a monoterapia con IFN- $\alpha$, se encontró una mediana de supervivencia 8,1 meses superior en el grupo que también fue tratado con nefrectomía ${ }^{38}$. En los pacientes con enfermedad metastásica la nefrectomía está indicada cuando los pacientes son susceptibles de cirugía y tienen un buen estado general de salud ${ }^{38,39}$. 


\subsection{Resección de las metástasis}

La resección completa de las metástasis puede contribuir a mejorar el pronóstico clínico. Cuando se consigue resecar completamente las metástasis o en recurrencias aisladas locales la inmunoterapia adyuvante no mejora el pronóstico clínico ${ }^{37}$. En pacientes con extensión metastásica sincrónica la metastasectomía debe realizarse en caso de enfermedad resecable y buen estado general, teniendo en cuenta que el pronóstico clínico es peor que en los pacientes con metástasis no sincrónicas.

\subsection{Radioterapia para las metástasis del CCR}

La radioterapia se puede utilizar en pacientes sintomáticos seleccionados con lesiones cerebrales $\mathrm{u}$ óseas irresecables que no han respondido a otros tratamientos conservadores ${ }^{40,41}$. En determinados casos la radioterapia de las lesiones cerebrales (irradiación de todo el cerebro o abordaje estereotáctico) y de las lesiones óseas puede inducir un alivio de los síntomas debidos al CCR metastásico ${ }^{41} 42$.

\subsection{Terapia sistémica para el CCR metastásico}

5.4.1 Quimioterapia

Debido a que la mayoría de los CCR se desarrollan a partir del túbulo proximal, tienen altos niveles de expresión de la proteína de resistencia a drogas P-glicoproteína y por ello son resistentes a la mayoría de los quimioterápicos. Parece que la quimioterapia sólo es eficaz si se asocia 5-fluorouracilo (5-FU) a otros inmunoterápicos, aunque los resultados aún están por publicar ${ }^{43}$. La quimioterapia no se recomienda en los pacientes con CCR metastásico.

\subsubsection{Inmunoterapia}

En estudios aleatorizados el IFN- $\alpha$ ha demostrado mejor supervivencia que la hormonoterapia en pacientes con CCR metastásico ${ }^{44}$. Los pacientes que se beneficiaron de este tratamiento tenían un buen estado general (estatus 0-1 según la OMS) y fueron tratados durante al menos 12 semanas y hasta 1 año, con una mejoría de la supervivencia de varios meses. La interleukina 2 (IL-2) se ha utilizado en pacientes con CCR metastásico desde 1985 con mayor toxicidad que el IFN- $\alpha$. Numerosos estudios han demostrado un rango de respuesta de entre $7 \%$ a $27 \%{ }^{45-47}$. No está claro el régimen óptimo de IL-2, pero los pacientes que han tenido una respuesta completa durante un largo período de tiempo (más de 10 años) han recibido una dosis elevada (bolus) ${ }^{48}$. Sin embargo, no se ha hecho ningún estudio aleatorizado comparado con tratamiento paliativo. Parece que sólo el cáncer renal de células claras responde a la inmunoterapia.

Se han realizado numerosos estudios aleatorizados para investigar acerca de la eficacia de la combinación de citocinas. La supervivencia de los pacientes no fue superior a la observada con monoterapia $^{49}$. Ninguna otra combinación utilizando ácido cis-retinoico ó 5-FU ha mostrado un beneficio clínico relevante ${ }^{50,51}$. En resumen, la inmunoterapia puede aportar un beneficio en algunos pacientes de bajo riesgo con cáncer renal metastásico de células claras.

\subsubsection{Fármacos inhibidores de la angiogénesis}

Los avances recientes en el conocimiento de la biología molecular del CCR han llevado al desarrollo de múltiples agentes novedosos para el tratamiento del CCR metastásico. En concreto, la inactivación VHL es un hecho frecuente en el CCR esporádico, que lleva a la acumulación de HIF y a la activación de genes hipoxia-inducibles como VEGF y PDGF, que son dianas de fármacos que inhiben la angiogénesis ${ }^{52,53}$. Recientemente dos fármacos inhibidores de la angiogénesis se han aprobado en los Estados Unidos de América y en Europa para el tratamiento del CCR metastásico: sorafenib (Nexavar®) y sunitinib (Sutent®).

Sorafenib es un fármaco oral que inhibe múltiples kinasas; tiene actividad contra Raf-1serine/ threonine kinase, B-Raf, VEGFR-2, PDGFR, FLT-3 y c-Kit. Un ensayo en fase 3 comparando sorafenib y placebo tras haber recibido, sin resultado, inmunoterapia previamente, mostró una mediana de supervivencia libre de progresión de 24 semanas para el sorafenib y de 12 semanas para el placebo ( $\mathrm{p}<0,000001)$. Tras 3 meses de tratamiento, el 75\% de los pacientes que recibieron sorafenib permanecieron sin progresión, frente al 43\% del grupo de placebo ${ }^{54}$. Aún no se tienen datos sobre la supervivencia global, aunque el uso cruzado de los inhibidores de la angiogénesis dificultará la interpretación de esta información. Sunitinib es un inhibidor de la oxindol tirosin kinasa (TK). Es una pequeña molécula con actividad antitumoral y antiangiogénica, que actúa frente a varias dianas como PDGFR. VEGFR, KIT y FLT-3. Dos ensayos multicéntricos en 
fase 2 con sunitinib como tratamiento de segunda línea en monoterapia en pacientes con cáncer renal metastásico demostró un tasa de respuesta parcial de 34-40\%; un 27-29\% de los pacientes presentaron una estabilización de la enfermedad durante $\geq 3$ meses 55,56 . Se ha publicado recientemente un ensayo en fase 3 comparando el sunitinib como primera línea en monoterapia frente a IFN- $\alpha$. La mediana de supervivencia libre de progresión fue superior en los pacientes tratados con sunitinib ( 11 meses) que en aquellos tratados con IFN- $\alpha$ (5 meses), $\mathrm{p}<0,000001$; esto sugiere que en los pacientes con CCR metastásico y riesgo bajo o medio, el tratamiento con IFN- $\alpha$ como primera línea es inferior en resultados que el sunitinib ${ }^{57}$. Los datos de supervivencia global derivados de este estudio todavía están por publicar, pero es llamativo que sólo el 6\% de los pacientes del grupo de IFN- $\alpha$ presentan respuesta.

Temsirolimus, que es un inhibidor específico de mTOR (mammalian target of rapamycin), es también un fármaco emergente importante para el CCR metastásico ${ }^{53}$. Recientemente se ha publicado un ensayo fase 3 comparando temsirolimus, IFN- $\alpha$ y su combinación en pacientes con CCR avanzado, como tratamiento de primera línea. Se ha demostrado que temsirolimus en monoterapia incrementa la supervivencia global en los pacientes de bajo riesgo, en comparación con el IFN- $\alpha$ o con la combinación de IFN- $\alpha$ y te, sirolimus ${ }^{58}$.

El papel exacto de los nuevos fármacos aún está abierto al debate; en el futuro se llevarán a cabo estudios de combinación. Actualmente no hay datos disponibles acerca de si curarán a algunos pacientes, o de si estabilizarán el CCR metastásico durante un periodo prolongado. Se debe valorar esto, teniendo en cuenta también la toxicidad de estos fármacos y su repercusión sobre la calidad de vida.

\section{Seguimiento tras la cirugia radical}

El seguimiento tras la cirugía radical permite al urólogo monitorizar o identificar las complicaciones postoperatorias, la función renal, la recurrencia local o en el riñón contralateral, y el desarrollo de metástasis. Las complicaciones y la función renal son evaluadas por la historia, el examen físico y las determinaciones de creatinina. Se indica controlar durante largos periodos de tiempo los niveles de creatinina si la función renal estaba dañada previamente a la cirugía o en el postoperatorio. La recu- rrencia local es rara $(1,8 \%)$, pero dado que el tratamiento más eficaz es la cirugía citorreductora, el diagnóstico precoz es de gran utilidad. La recurrencia en el riñón contralateral también es poco frecuente (2-3\%) y se relaciona con márgenes quirúrgicos positivos, multifocalidad, y el grado. Una de las justificaciones del seguimiento tras la cirugía es para identificar precozmente las metástasis, de modo que sea factible la resección quirúrgica. Además, un temprano diagnóstico de la recurrencia mejora la eficacia del tratamiento sistémico, ya que la carga tumoral es menor.

No es necesario realizar un seguimiento radiológico exhaustivo en los pacientes con tumores pequeños bien diferenciados, ya que los resultados tras la cirugía en este tipo de tumores suele ser excelente. Por lo tanto, es razonable ajustar el seguimiento de acuerdo con el riesgo de desarrollar una recurrencia o de metástasis ${ }^{59}$.

Cuando la probabilidad de recurrencia es baja es suficiente con la realización de radiografías de tórax y ecografías. Cuando el riesgo es intermedio o alto es de elección la TC toracoabdominal, teniendo en cuenta que la radiación de las repetidas pruebas es elevada. Otra cuestión es la duración óptima del seguimiento. Algunos consideran que el seguimiento, pasado 5 años, deja de ser coste efectivo; sin embargo, las metástasis de aparición tardía suelen ser solitarias, justificando un tratamiento agresivo con intención curativa. Los pacientes que desarrollan un tumor en el riñón contralateral pueden ser tratados mediante cirugía conservadora del órgano si se detectan precozmente. Para tumores menores de $4 \mathrm{~cm}$ no existe diferencia en cuanto a recurrencia tras la nefrectomía parcial o radical ${ }^{60}$.

Utilizando muchos de estos parámetros varios grupos han diseñado sistemas de puntuación $\mathrm{y}$ algoritmos para estratificar a los pacientes en grupos de riesgo bajo, intermedio y alto, en cuanto a desarrollo de recurrencia o metástasis se refiere. Las frecuencias y tipo de investigación son diferentes para cada grupo ${ }^{61-64}$. La Tabla 2 recoge un ejemplo de estos sistemas de gradación y la Tabla 3 muestra el riesgo acumulado de desarrollo de metástasis (\%) tras la nefrectomía en pacientes con CCR de células claras, según la Mayo Scoring System $^{64}$. El uso de estos sistemas de gradación permite a los urólogos ser selectivos en la utilización de técnicas de imagen, así como detectar a los pacientes que requieren un seguimiento más estrecho. 
Tabla 2. Algoritmo para predecir el desarrollo de metástasis tras la nefrectomía en pacientes con CCR de células claras de acuerdo con el Mayo Scoring System ${ }^{62}$

\begin{tabular}{|c|c|c|c|c|}
\hline \multicolumn{3}{|c|}{ Característica } & \multicolumn{2}{|c|}{ Puntuación } \\
\hline \multicolumn{5}{|c|}{ Estadio $\mathrm{T}$ del tumor primario } \\
\hline \multicolumn{3}{|l|}{ Tla } & & 0 \\
\hline \multicolumn{3}{|l|}{ pT1b } & & 2 \\
\hline \multicolumn{3}{|l|}{ pT2 } & & 3 \\
\hline \multicolumn{3}{|l|}{ pT3-pT4 } & & 4 \\
\hline \\
\hline \multicolumn{3}{|c|}{$\begin{array}{l}\text { Tamaño tumoral } \\
<10 \mathrm{~cm}\end{array}$} & & 0 \\
\hline \multicolumn{3}{|l|}{$>10 \mathrm{~cm}$} & & 1 \\
\hline \multicolumn{5}{|c|}{ Estado de los ganglios regionales } \\
\hline \multicolumn{3}{|l|}{$\mathrm{pNx} / \mathrm{pNO}$} & & 0 \\
\hline \multicolumn{3}{|c|}{ pN1-pN2 } & & 2 \\
\hline \multicolumn{5}{|c|}{ Grado nuclear } \\
\hline \multicolumn{3}{|c|}{ Grado $1-2$} & & 0 \\
\hline \multicolumn{3}{|l|}{ Grado 3} & & 1 \\
\hline \multicolumn{3}{|l|}{ Grado 4} & & 3 \\
\hline \multicolumn{5}{|c|}{ Necrosis tumoral } \\
\hline \multicolumn{3}{|c|}{ No necrosis } & & 0 \\
\hline \multicolumn{3}{|l|}{ Necrosis } & & 1 \\
\hline \multicolumn{5}{|c|}{$\begin{array}{l}\text { Los grupos de riesgo se pueden estratificar por medio de este siste- } \\
\text { ma en riesgo bajo 0-2, riesgo intermedio } 3-5 \text { y riesgo elevado }>6 \text {, de } \\
\text { acuerdo con la Mayo Scoring System }{ }^{62} \text {. }\end{array}$} \\
\hline \multicolumn{5}{|c|}{$\begin{array}{l}\text { Tabla 3. Riesgo acumulado de desarrollo de metástasis } \\
(\%) \text { tras la nefrectomía en pacientes con CCR de células } \\
\text { claras en función de los grupos definidos por la Mayo } \\
\text { Scoring System } 62\end{array}$} \\
\hline $\begin{array}{l}\text { Grupo de } \\
\text { riesgo }\end{array}$ & $\begin{array}{c}\text { Primer } \\
\text { año }\end{array}$ & $\begin{array}{c}\text { Tercer } \\
\text { año }\end{array}$ & $\begin{array}{c}\text { Quinto } \\
\text { año }\end{array}$ & $\begin{array}{c}\text { Décimo } \\
\text { año }\end{array}$ \\
\hline Bajo & 0,5 & 2,1 & 2,9 & 7,5 \\
\hline Intermedio & 9,6 & 20,2 & 26,2 & 35,7 \\
\hline Alto & 42,3 & 62,9 & 68,8 & 76,4 \\
\hline
\end{tabular}

Cuando el riesgo de recurrencia tumoral o de progresión sistémica es muy bajo, no es necesario realizar TC. En el grupo de riesgo intermedio se debe realizar un seguimiento más estrecho, que incluye TC periódicamente. En los pacientes de alto riesgo el seguimiento incluye rutinariamente TC. Por tanto, la intensidad de los programas de seguimiento para un paciente concreto se debe adaptar en función del riesgo de recurrencia tumoral o de progresión sistémica del tumor, según las directrices de los diferentes nomogramas diseñados para tal fin.

\section{Conflicto de intereses}

No hay conflicto de intereses comerciales en esta revisión.

\section{REFERENCIAS}

1. European Network of Cancer Registries. Eurocim version 4.0. European incidence database V2.3, 730 entity dictionary (2001). Lyon, France, 2001.

2. Lindblad P. Epidemiology of renal cell carcinoma. Scand J Surg 2004;93(2):88-96.

3. Kovacs G, Akhtar M, Beckwith BJ, Bugert P, Cooper CS, Delahunt B, Eble JN, Fleming S, Ljungberg B, Medeiros LJ, Moch H, Reuter VE, Ritz E, Roos G, Schmidt D, Srigley JR, Störkel S, van den Berg E, Zbar B. The Heidelberg classification of renal cell tumours. J Pathol. 1997;183(2):131-133.

4. Pischon T, Lahmann PH, Boeing H, Tjønneland A, Halkjaer J, Overvad $\mathrm{K}$, et al. Body size and risk of renal cell carcinoma in the European Prospective Investigation into Cancer and Nutrition (EPIC). Int J Cancer. 2006;118(3):728-738.

5. Patard JJ, Rodriguez A, Rioux-Leclercq N, Guillé F, Lobel B. Prognostic significance of the mode of detection in renal tumours. BJU Int. 2002;90(4):358-363.

6. Kato M, Suzuki T, Suzuki Y, Terasawa Y, Sasano H, Arai Y. Natural history of small renal cell carcinoma: evaluation of growth rate, histological grade, cell proliferation and apoptosis. J Urol. 2004;172(3):863-866.

7. Novick AC, Campbell SC. Renal tumours. In: Walsh PC, Retik $\mathrm{AB}$, Vaughan ED, Wein AJ, editors. Campbell's urology. Philadelphia: WB Saunders; 2002. p. 2672-731.]

8. Lee CT, Katz J, Fearn PA, Russo P. Mode of presentation of renal cell carcinoma provides prognostic information. Urol Oncol. 2002;7(4):135-40.

9. Patard JJ, Leray E, Rodriguez A, Rioux-Leclercq N, Guillé F, Lobel B. Correlation between symptom graduation, tumor characteristics and survival in renal cell carcinoma. Eur Urol. 2003;44(2):226-232.

10. Sufrin G, Chasan S, Golio A, Murphy GP. Paraneoplastic and serologic syndromes of renal adenocarcinoma. Semin Urol. 1989;7(3):158-171.

11. Heidenreich A, Ravery V; European Society of Oncological Urology. Preoperative imaging in renal cell cancer. World J Urol. 2004;22(5):307-315.

12. Hricak H, Demas BE, Williams RD, McNamara MT, Hedgcock MW, Amparo EG, et al. Magnetic resonance imaging in the diagnosis and staging of renal and perirenal neoplasms. Radiology. 1985;154(3):709-715.

13. Gupta NP, Ansari MS, Khaitan A, Sivaramakrishna MS, Hemal AK, Dogra PN, et al. Impact of imaging and thrombus level in management of renal cell carcinoma extending to veins. Urol Int. 2004;72(2): 129-134.

14. Sobin LH. The WHO histological classification of urinary bladder tumours. Urol Res. 1978;6(4):193-195.

15. Ficarra V, Schips L, Guillè F, Li G, De La Taille A, Prayer Galetti T, et al. Multiinstitutional European validation of the 2002 TNM staging system in conventional and papillary localized renal cell carcinoma. Cancer. 2005 Sep 1;104(5):968-974.

16. Thompson RH, Leibovich BC, Cheville JC, Webster WS, Lohse $\mathrm{CM}$, Kwon ED, et al. Is renal sinus fat invasion the same as perinephric fat invasion for pT3a renal cell carcinoma?. J Urol. 2005;174(4 Pt 1):1218-1221.

17. Han KR, Bui MH, Pantuck AJ, Freitas DG, Leibovich BC, Dorey FJ, et al. TNM T3a renal cell carcinoma: adrenal gland involvement is not the same as renal fat invasion. J Urol. 2003;169 (3):899-903.

18. Terrone C, Cracco C, Porpiglia F, Bollito E, Scoffone C, Poggio $\mathrm{M}$, et al. Reassessing the current TNM lymph node staging for renal cell carcinoma. Eur Urol. 2006;49(2):324-331.

19. Eble JN, Sauter G, Epstein JI, Sesterhenn IA, editors. Pathology and genetics of tumours of the urinary system and male genital organs. World Health Organization Classification of Tumours. Lyon, France: IARC Press; 2004. p7. 
20. Delahunt B, Eble JN, McCredie MR, Bethwaite PB, Stewart JH, Bilous AM. Morphologic typing of papillary renal cell carcinoma: comparison of growth kinetics and patient survival in 66 cases. Hum Pathol. 2001;32(6):590-595.

21. Zhao H, Ljungberg B, Grankvist K, Rasmuson T, Tibshirani R, Brooks JD. Gene expression profiling predicts survival in conventional renal cell carcinoma. PLoS Med. 2006;3(1):e13.

22. Robson CJ, Churchill BM, Anderson W. The results of radical nephrectomy for renal cell carcinoma. 1969. J Urol 2002;167(2 Pt 2):873-875.

23. Kuczyk M, Münch T, Machtens S, Bokemeyer C, Wefer A, Hartmann J, et al. The need for routine adrenalectomy during surgical treatment for renal cell cancer: the Hannover experience. BJU Int. 2002;89(6):517-522.

24. Kuczyk M, Wegener G, Jonas U. The therapeutic value of adrenalectomy in case of solitary metastatic spread originating from primary renal cell cancer. Eur Urol. 2005;48(2):252-257 .

25. Glazer AA, Novick AC. Long-term followup after surgical treatment for renal cell carcinoma extending into the right atrium. J Urol. 1996;155(2):448-450.

26. Munro NP, Woodhams S, Nawrocki JD, Fletcher MS, Thomas PJ. The role of transarterial embolization in the treatment of renal cell carcinoma. BJU Int. 2003;92(3):240-244 .

27. Hafez KS, Fergany AF, Novick AC. Nephron sparing surgery for localized renal cell carcinoma: impact of tumor size on patient survival, tumor recurrence and TNM staging. $J$ Urol. 1999;162(6):1930-1933.

28. McKiernan J, Yossepowitch O, Kattan MW, Simmons R, Motzer RJ, Reuter VE, et al. Partial nephrectomy for renal cortical tumors: pathologic findings and impact on outcome. Urology. 2002;60(6): 1003-1009.

29. Link RE, Bhayani SB, Allaf ME, Varkarakis I, Inagaki T, Rogers $\mathrm{C}$, et al. Exploring the learning curve, pathological outcomes and perioperative morbidity of laparoscopic partial nephrectomy performed for renal mass. J Urol. 2005;173(5):1690-1694

30. Novick AC. Laparoscopic and partial nephrectomy. Clin Cancer Res. 2004;10(18 Pt 2):6322S-6327S.

31. Becker F, Siemer S, Humke U, Hack M, Ziegler M, Stöckle M Elective nephron sparing surgery should become standard treatment for small unilateral renal cell carcinoma: Long-term survival data of 216 patients. Eur Urol. 2006;49(2):308-313.

32. Ono Y, Hattori R, Gotoh M, Yoshino Y, Yoshikawa Y, Kamihira O. Laparoscopic radical nephrectomy for renal cell carcinoma: the standard of care already?. Curr Opin Urol. 2005;15(2):7578.

33. Matin SF, Gill IS, Worley S, Novick AC. Outcome of laparoscopic radical and open partial nephrectomy for the sporadic $4 \mathrm{~cm}$. or less renal tumor with a normal contralateral kidney. J Urol. 2002;168(4 Pt 1):1356-1359.

34. Lewin JS, Nour SG, Connell CF, Sulman A, Duerk JL, Resnick MI, et al. Phase II clinical trial of interactive MR imaging-guided interstitial radiofrequency thermal ablation of primary kidney tumors: initial experience. Radiology. 2004;232(3):835-845.

35. Gill IS, Remer EM, Hasan WA, Strzempkowski B, Spaliviero M, Steinberg AP, et al. Renal cryoablation: outcome at 3 years. J Urol. 2005;173(6):1903-1907.

36. Jocham D, Richter A, Hoffmann L, Iwig K, Fahlenkamp D, Zakrzewski G, et al. Adjuvant autologous renal tumour cell vaccine and risk of tumour progression in patients with renalcell carcinoma after radical nephrectomy: phase III, randomised controlled trial. Lancet. 2004;363(9409):594-599.

37. Flanigan RC, Mickisch G, Sylvester R, Tangen C, Van Poppel H, Crawford ED. Cytoreductive nephrectomy in patients with metastatic renal cancer: a combined analysis. J Urol. 2004;171 (3): 1071-1076.
38. Ljungberg B, Landberg G, Alamdari FI. Factors of importance for prediction of survival in patients with metastatic renal cell carcinoma, treated with or without nephrectomy. Scand J Urol Nephrol. 2000;34(4):246-251.

39. Van der Poel HG, Roukema JA, Horenblas S, van Geel AN, Debruyne FM. Metastasectomy in renal cell carcinoma: A multicenter retrospective analysis. Eur Urol.1999;35(3):197-203.

40. Fosså SD, Kjølseth I, Lund G. Radiotherapy of metastases from renal cancer. Eur Urol. 1982;8(6):340-342.

41. Andrews DW, Scott CB, Sperduto PW, Flanders AE, Gaspar LE, Schell MC, et al. Whole brain radiation therapy with or without stereotactic radiosurgery boost for patients with one to three brain metastases: phase III results of the RTOG 9508 randomised trial. Lancet. 2004;363(9422):1665-1672.

42. Kavolius JP, Mastorakos DP, Pavlovich C, Russo P, Burt ME, Brady MS. Resection of metastatic renal cell carcinoma. J Clin Oncol. 1998;16(6):2261-266.

43. Stadler WM, Huo D, George C, Yang X, Ryan CW, Karrison T, et al. Prognostic factors for survival with gemcitabine plus 5fluorouracil based regimens for metastatic renal cancer. J Urol. 2003;170(4 Pt 1):1141-1145.

44. Interferon-alpha and survival in metastatic renal carcinoma: early results of a randomised controlled trial. Medical Research Council Renal Cancer Collaborators. Lancet, 1999(353):14-17.

45. Rosenberg SA, Lotze MT, Yang JC, Topalian SL, Chang AE, Schwartzentruber DJ, Aebersold P, Leitman S, Linehan WM, Seipp CA, et al: Prospective randomized trial of high-dose interleukin-2 alone or in conjunction with lymphokine-activated killer cells for the treatment of patients with advanced cancer. JNatl Cancer Inst. 1993;85(8):622-632.

46. Fyfe G, Fisher RI, Rosenberg SA, Sznol M, Parkinson DR, Louie AC. Results of treatment of 255 patients with metastatic renal cell carcinoma who received high-dose recombinant interleukin-2 therapy. J Clin Oncol. 1995 Mar;13(3):688-696.

47. McDermott DF, Regan MM, Clark JI, Flaherty LE, Weiss GR, Logan TF, Kirkwood JM, Gordon MS, Sosman JA, Ernstoff MS, Tretter CP, Urba WJ, Smith JW, Margolin KA, Mier JW, Gollob JA, Dutcher JP, Atkins MB.: Randomized phase III trial of highdose interleukin-2 versus subcutaneous interleukin-2 and interferon in patients with metastatic renal cell carcinoma. J Clin Oncol. 2005 ;23(1):133-141.

48. Yang JC, Sherry RM, Steinberg SM, Topalian SL, Schwartzentruber DJ, Hwu P, Seipp CA, Rogers-Freezer L, Morton KE, White DE, Liewehr DJ, Merino MJ, Rosenberg SA. Randomized study of high-dose and low-dose interleukin-2 in patients with metastatic renal cancer. J Clin Oncol. 2003;21(16):3127-3132.

49. Negrier S, Escudier B, Lasset C, Douillard JY, Savary J, Chevreau C, Ravaud A, Mercatello A, Peny J, Mousseau M, Philip T, Tursz T.: Recombinant human interleukin-2, recombinant human interferon alfa-2a, or both in metastatic renalcell carcinoma. Groupe Francais d'Immunotherapie. N Engl J Med. 1998 Apr 30;338(18):1272-1278.

50. Aass N, De Mulder PH, Mickisch GH, Mulders P, van Oosterom AT, van Poppel H, Fossa SD, de Prijck L, Sylvester RJ. Randomized phase II/III trial of interferon Alfa-2a with and without 13-cis-retinoic acid in patients with progressive metastatic renal cell Carcinoma: the European Organisation for Research and Treatment of Cancer Genito-Urinary Tract Cancer Group (EORTC 30951). J Clin Oncol. 2005 Jun 20;23 (18):4172-4178.

51. Atzpodien J, Kirchner H, Illiger HJ, Metzner B, Ukena D, Schott H, Funke PJ, Gramatzki M, Jürgenson S, Wandert T, Patzelt T, Reitz M.: IL-2 in combination with IFN- alpha and 5-FU versus tamoxifen in metastatic renal cell carcinoma: long-term results of a controlled randomized clinical trial. Br J Cancer. 2001 Oct 19;85(8):1130-1136. 
52. Patard JJ, Rioux-Leclercq N, Fergelot P. Understanding the importance of smart drugs in renal cell carcinoma. Eur Urol. 2006 Apr;49(4):633-643.

53. Patel PH, Chadalavada RS, Chaganti RS, Motzer RJ. Targeting von Hippel-Lindau pathway in renal cell carcinoma. Clin Cancer Res. 2006;12(24):7215-7120.

54. Escudier B, Eisen T, Stadler WM, Szczylik C, Oudard S, Siebels M, Negrier S, Chevreau C, Solska E, Desai AA, Rolland F, Demkow T, Hutson TE, Gore M, Freeman S, Schwartz B, Shan M, Simantov R, Bukowski RM; TARGET Study Group.: Sorafenib in advanced clear-cell renal-cell carcinoma. N Engl J Med. 2007;356(2):125-134.

55. Motzer RJ, Rini BI, Bukowski RM, Curti BD, George DJ, Hudes GR, Redman BG, Margolin KA, Merchan JR, Wilding G, Ginsberg MS, Bacik J, Kim ST, Baum CM, Michaelson MD: Sunitinib in patients with metastatic renal cell carcinoma. JAMA. 2006 Jun 7;295(21):2516-2524.

56. Motzer RJ, Michaelson MD, Redman BG, Hudes GR, Wilding G, Figlin RA, Ginsberg MS, Kim ST, Baum CM, DePrimo SE, Li JZ, Bello CL, Theuer CP, George DJ, Rini BI. Activity of SU11248, a multitargeted inhibitor of vascular endothelial growth factor receptor and platelet-derived growth factor receptor, in patients with metastatic renal cell carcinoma. J Clin Oncol. 2006 Jan 1;24(1):16-24.

57. Motzer RJ, Hutson TE, Tomczak P, Michaelson MD, Bukowski RM, Rixe O, Oudard S, Negrier S, Szczylik C, Kim ST, Chen I, Bycott PW, Baum CM, Figlin RA.: Sunitinib versus interferon alfa in metastatic renal-cell carcinoma. N Engl J Med. 2007 Jan 11;356(2): 115-124.

58. Hudes G, Carducci M, Tomczak P, et al. A phase 3 randomized, 3-arm study of temsirolimus (TEMSR) or interferon-alpha (IFN) or the combination of TEMSR + IFN in the treatment of firstline, poor-risk patients with advanced renal cell carcinoma (adv RCC). J Clin Oncol (Meeting Abstracts) 2006;24:LBA4.

59. Lam JS, Shvarts O, Leppert JT, Figlin RA, Belldegrun AS. Renal cell carcinoma 2005: new frontiers in staging, prognostication and targeted molecular therapy. J Urol. 2005;173(6):18531862 .
60. Patard JJ, Shvarts O, Lam JS, Pantuck AJ, Kim HL, Ficarra V. Safety and efficacy of partial nephrectomy for all T1 tumors based on an international multicenter experience. J Urol,. 2004;171(6 Pt 1):2181-2435.

61. Ljungberg B, Alamdari FI, Rasmuson T, Roos G. Follow-up guidelines for nonmetastatic renal cell carcinoma based on the occurrence of metastases after radical nephrectomy. BJU Int. 1999;84(4):405-411.

62. Kattan MW, Reuter V, Motzer RJ, Katz J, Russo P. A postoperative prognostic nomogram for renal cell carcinoma. J Urol. 2001;166(1):63-67.

63. Lam JS, Shvarts O, Leppert JT, Pantuck AJ, Figlin RA, Belldegrun AS, et al. Postoperative surveillance protocol for patients with localized and locally advanced renal cell carcinoma based on a validated prognostic nomogram and risk group stratification system. J Urol. 2005;174(2): 466-472.

64. Leibovich BC, Blute ML, Cheville JC, Lohse CM, Frank I, Kwon ED, et al. Prediction of progression after radical nephrectomy for patients with clear cell renal cell carcinoma: a stratification tool for prospective clinical trials. Cancer2003;97(7): 16631671.

Autor para correspondencia: Börje Ljungberg

Department of Surgical and Perioperative Sciences, Urology and Andrology, Umea ${ }^{\circ}$ University, S-901 85 Umea $^{\circ}$, Sweden. Tel. +4690785 1330

Fax: +4690 125396 .

E-mail address: borje.ljungberg@urologi.umu.se (B. Ljungberg).

Correspondencia autor traducción: Dr. Gonzalo García Fadrique Servicio de Urología

Hospital Universitario La Fe

Avda. Campanar, 21 - 46009 Valencia

Tel.: 963862700

E-mail autor: gonzalogfadrique@comv.es

Información artículo: Revisión - Cáncer de próstata 